\title{
Electromagnetic Scattering by a Moving Conducting Sphere'
}

\author{
Robert C. Restrick, III \\ University of Michigan, Radiation Laboratory, Department of Electrical Engineering, \\ Ann Arbor, Mich. 48108, U.S.A. \\ (Received March 11, 1968; revised July 9, 1968)
}

\begin{abstract}
A relativistically correct expression for the far zone electromagnetic field scattered by a perfectly conducting sphere, moving with constant velocity, through an incident plane wave is developed. It is seen that if the scattered field is expressed in a special "retarded" coordinate system, a result that is more tractible, allowing physical interpretation, is obtained. When the field is expressed in this way, the expected first-order effects are manifest, yet the higher order effects are also present. This solution may be directly applied to the finite bodies with more complicated shapes than the sphere. For the case of the sphere it is found that the form of the scattered field allows the calculation of the scattering cross section, the total scattering cross section, and the rate of exchange of electromagnetic and mechanical energies.
\end{abstract}

\section{Introduction}

The Special Theory of Relativity gives a rigorous means of discussing a situation involving electromagnetic scattering by a uniformly moving body: For such a situation, in free space, the theory specifies how one may restate the problem so that it is viewed with respect to an observer moving with the body. From this viewpoint the problem appears that of scattering by a stationary body. This implies that there exists a straightforward means of obtaining the field scattered by a uniformly moving body in free space, providing only that its stationary scattering properties are known.

Historically, the first situation attacked by this method was that of reflection by a moving infinite mirror (Sommerfeld, 1964b; Pauli, 1958). Scattering by a moving dielectric half space also has been discussed by Tai (1965), Yeh (1965), and by Pyati (1966). Their work has been extended to a moving uniaxially anisotropic half space by Lee and Lo (1967). Recently, Lee and Mittra (1967) have considered the case of scattering by a moving conducting cylinder.

It is seen that as the geometry of the scattering body becomes more complex, the original symmetry of the stationary solution is lost in the transformation between the moving systems, resulting in an expression that is awkward, yielding little physical meaning.

In this paper a coordinate system is chosen to express the far field scattered by a moving finite body, a sphere, giving a solution that is relativistically correct and at the same time allows physical interpretation. Although a sphere is considered here, the method may be used to express the far field scattered by other moving finite objects or the radiation field from a finite, moving electromagnetic source.

\section{The Problem in the Rest Frame}

The problem consists of a uniformly moving, perfectly conducting sphere with radius $a$ and velocity $\bar{v}$, scattering a plane wave propagating in free space with propagation vector $\bar{k}$ and magnitude $E^{(i)}$. Here, two four-dimensional (three spatial and one temporal) coordinate systems will be used - the one in which the problem is given, where the sphere is seen to be moving, to be called

${ }^{1}$ The research reported in this paper was sponsored by the National Science Foundation under grant GK-1213. 
the rest frame, and the one moving with the sphere, in which the sphere appears stationally, to be called the moving frame.

In each frame there will exist two physically important directions - the direction of motion and the direction of propagation of the incident wave. A spatial coordinate system will be associated with each of these directions. Those in the rest frame are shown in figure 1 . The $x, y, z$ Cartesian spatial coordinate system is chosen so that the motion of the sphere is given by $x=0, y=0$, and $z=v t$. The incident plane wave is specified by the $\xi, \eta, \zeta$ system. Its direction of propagation is the negative $\zeta$ direction and its polarization is designated by the angle $\psi$ between the $\eta$ axis and the $\bar{E}$ vector. No generality is lost by making the $y$ and $\eta$ axis common so that the transformation between the $x, y, z$ system and $\xi, \eta, \zeta$ system is a rotation about the common $y$ or $\eta$ axis through the angle of incidence, $\theta^{(i)}$.

The incident field may be expressed

$$
\begin{gathered}
\bar{E}^{(i)}=\frac{E^{(i)}}{2}\{\cos \psi \hat{\eta}-\sin \psi \hat{\xi}\} e^{-i(k \zeta+\omega t)}+c . c . \\
c \bar{B}^{(i)}=-\hat{\zeta} \dot{\times} \bar{E}^{(i)}
\end{gathered}
$$

Here the fields are written as real quantities by adding to each complex expression its complex conjugate, c.c. The symbols $\hat{\xi}, \hat{\eta}$, and $\hat{\zeta}$ represent unit vectors in the $\xi, \eta$, and $\zeta$ directions respectively.

\section{The Problem in the Moving Frame}

The entire problem may now be transformed to the moving frame; where all quantities will be denoted by primes. Here two rectangular spatial systems, the $x^{\prime}, y^{\prime}, z^{\prime}$ and $\xi^{\prime}, \eta^{\prime}, \zeta^{\prime}$ systems, will be defined in a manner analogous to those previously defined in the rest frame. Following the prescribed rules for transformation of the various physical quantities (see for instance Sommerfeld, 1964a) the problem in the moving system consists of a stationary sphere, located in the origin, scattering a plane wave given by ${ }^{2}$

$$
\begin{aligned}
& \bar{E}^{\prime(i)}=\frac{E^{(i)}}{2}\left(\frac{1+\beta \cos \theta^{(i)}}{\sqrt{1-\beta^{2}}}\right)\left\{\cos \psi \hat{\eta}^{\prime}-\sin \psi \hat{\xi}^{\prime}\right\} e^{-i\left(k^{\prime} \zeta^{\prime}+\omega^{\prime} t^{\prime}\right)}+\text { c.c. }, \\
& c \bar{B}^{\prime(i)}=-\hat{\zeta}^{\prime} \times \bar{E}^{\prime(i)},
\end{aligned}
$$

where

$$
\beta=v / c ; \omega^{\prime}=\omega \frac{1+\beta \cos \theta^{(i)}}{\sqrt{1-\beta^{2}}}, \text { and } k^{\prime}=\frac{1+\beta \cos \theta^{(i)}}{\sqrt{1-\beta^{2}}} k
$$

Here the angle of incidence, $\theta^{\prime(i)}$, between the $\zeta^{\prime}$ axis and the $z^{\prime}$ axis is given by the relation

$$
\cos \theta^{\prime(i)}=\frac{\cos \theta^{(i)}+\beta}{1+\beta \cos \theta^{(i)}}
$$

Let the spherical coordinate system $R^{\prime}, \theta^{\prime}, \phi^{\prime}$ be defined so that the polar axis is the $\zeta^{\prime}$ axis and the dihedral angle $\phi^{\prime}$ is measured from the $\eta^{\prime}-\zeta^{\prime}$ plane. In the moving frame, the solution is the well-known Mie solution. Only the far-zone solution $\left(k^{\prime} R^{\prime} \gg 1\right)$ is considered here, which is

$$
\begin{aligned}
\vec{E}^{\prime}(s)= & -i \frac{E^{(i)}}{2}\left(\frac{1+\beta \cos \theta^{(i)}}{\sqrt{1-\beta^{2}}}\right)\left\{\cos \left(\phi^{\prime}-\psi\right) S_{1}\left(\theta^{\prime}\right) \hat{\theta}^{\prime}\right. \\
& \left.-\sin \left(\theta^{\prime}-\psi\right) S_{2}\left(\theta^{\prime}\right) \hat{\phi}^{\prime}\right\} \frac{e^{i k^{\prime}\left(R^{\prime}-c t^{\prime}\right)}}{k^{\prime} R^{\prime}}+c . c . \\
c \bar{B}^{\prime(s)}= & \hat{R}^{\prime} \times \bar{E}^{\prime(s)}
\end{aligned}
$$

2 The fact that a plane wave transforms to a plane wave with all new parameters (frequency, magnitude, direction of propagation, etc.) except polarization angle $\psi$ was obtained by direct application of the Special Theory of Relativity. It is not obvious why the angle $\psi$ should remain unchanged. 


$$
\begin{aligned}
& S_{1}\left(\theta^{\prime}\right)=\sum_{n=1}^{\infty} \frac{2 n+1}{n(n+1)}(-1)^{n}\left(\beta_{n} \frac{d P_{n}^{(1)}\left(\cos \theta^{\prime}\right)}{d \theta^{\prime}}-\alpha_{n} \frac{P_{n}^{(1)}\left(\cos \theta^{\prime}\right)}{\sin \theta^{\prime}}\right) \\
& S_{2}\left(\theta^{\prime}\right)=\sum_{n=1}^{\infty} \frac{2 n+1}{n(n+1)}(-1)^{n}\left(\beta_{n} \frac{P_{n}^{(1)}\left(\cos \theta^{\prime}\right)}{\sin \theta^{\prime}}-\alpha_{n} \frac{d P_{n}^{(1)}\left(\cos \theta^{\prime}\right)}{d \theta^{\prime}}\right)
\end{aligned}
$$

In these equations, $P_{n}^{(1)}$ is the associated Legendre function of the first kind,

$$
\alpha_{n}=\left.\frac{j_{n}(u)}{h_{n}^{(1)}(u)}\right|_{u=k^{\prime} a} \text { and } \beta_{n}=\left.\frac{\frac{d}{d u} u j_{n}(u)}{\frac{d}{d u} u h_{n}^{(1)}(u)}\right|_{u=k^{\prime} a} \text {, }
$$

and $h_{n}^{(1)}$ and $j_{n}$ are spherical Bessel functions.

\section{The Behavior of the Scattered Field in the Rest Frame}

It is well known that the far-field expressions of the type seen in (7) have their behavior dominated by the exponential function. Let it, $\exp \left[i k^{\prime}\left(R^{\prime}-c t^{\prime}\right)\right]$, be examined separately. This is done by using the Lorentz transformation to express the exponent of (7) in terms of the unprimed variables, and equating the result to a constant depending on a quantity $\tau$, which will be seen to have physical meaning. That is

$$
i k^{\prime}\left(R^{\prime}-c t^{\prime}\right)=i k^{\prime}\left\{\left[x^{2}+y^{2}+\left(\frac{z-v t}{\sqrt{1-\beta^{2}}}\right)^{2}\right]^{1 / 2}-c\left(\frac{t-\left(v z / c^{2}\right)}{\sqrt{1-\beta^{2}}}\right)\right\}=-i k^{\prime} c \tau \sqrt{1-\beta^{2}}
$$

Equation (11) is squared and the result simplified to give the following equation:

$$
x^{2}+y^{2}+(z-v \tau)^{2}=c^{2}(t-\tau)^{2} .
$$

This represents a spherical wave front propagating with the velocity of light. The wave front appears to have been emitted at the time $t=\tau$ at which time the sphere's position was $x=0$, $y=0, z=v \tau$.

As $\tau$ goes from $\tau_{1}$ to $\tau_{2}$ such that

$$
\tau_{2}-\tau_{1}=\frac{2 \pi}{k^{\prime} c \sqrt{1-\beta^{2}}}
$$

the exponent of (7) changes by $2 \pi$ and the function goes through one period of oscillation.

An effective wavelength $\lambda_{e}$ may be defined as the distance between two waves fronts emitted respectively at $\tau_{1}$ and $\tau_{2}$. Let an observer's position be given by the angle $\theta$ shown in figure 2 . Then using the far-field assumption

$$
\begin{aligned}
\lambda_{e} & =c\left(t-\tau_{1}\right)-c\left(t-\tau_{2}\right)-v\left(\tau_{2}-\tau_{1}\right) \cos \theta \\
& =\frac{2 \pi(1-\beta \operatorname{vos} \theta)}{k^{\prime} \sqrt{1-\beta^{2}}} .
\end{aligned}
$$

Associated with this will be an effective scattered frequency $\omega^{(s)}$ and wave number $k^{(s)}$ where

$$
\omega^{(s)}=\frac{2 \pi c}{\lambda_{e}}=\omega \frac{1+\beta \cos \theta^{(i)}}{1-\beta \cos \theta}
$$

and

$$
k^{(s)}=\omega^{(s)} / c \text {. }
$$


Strictly speaking the behavior is spatially and temporally harmonic only if $\Theta$ equals 0 or $\pi$. Elsewhere such quantities as wavelength and frequency are only approximations valid over a certain interval of time and a certain volume of space.

\section{The Transformation to the Rest Frame}

By direct substitution it is seen that the function $\tau(x, y, z, t)$ defined by (12) satisfies the relation

$$
\left(\frac{\partial \tau}{\partial x}\right)^{2}+\left(\frac{\partial \tau}{\partial y}\right)^{2}+\left(\frac{\partial \tau}{\partial z}\right)^{2}-\left(\frac{\partial \tau}{\partial c t}\right)^{2}=0
$$

implying that the surfaces,

$$
\tau(x, y, z, c t)=\tau_{0},
$$

where $\tau_{0}$ is constant, are characteristics of the hyperbolic partial differential equation (Courant and Hilbert, 1962). Alternately, for an observer at the position $x, y, z$, and time $t$, $v \tau$ would correspond to the retarded position of the sphere and $\tau$ the retarded time. It appears that the function $\tau$ is closely connected with this problem, and a way of expressing the scattered field by means of it will now be presented (Lewis and Pressman, 1966).

In each of the four rectangular coordinate systems (two in the rest frame, two in the moving frame) a spherical coordinate system will be defined as follows:

(i) The $R^{\prime}, \theta^{\prime}, \phi^{\prime}, t^{\prime}$ system and the $\xi^{\prime}, \eta^{\prime}, \zeta^{\prime}, t^{\prime}$ system

$$
\begin{aligned}
\zeta^{\prime} & =R^{\prime} \cos \theta^{\prime} \\
\eta^{\prime} & =R^{\prime} \sin \theta^{\prime} \cos \phi^{\prime} \\
\xi^{\prime} & =-R^{\prime} \sin \theta^{\prime} \sin \phi^{\prime} \\
t^{\prime} & =t^{\prime} .
\end{aligned}
$$

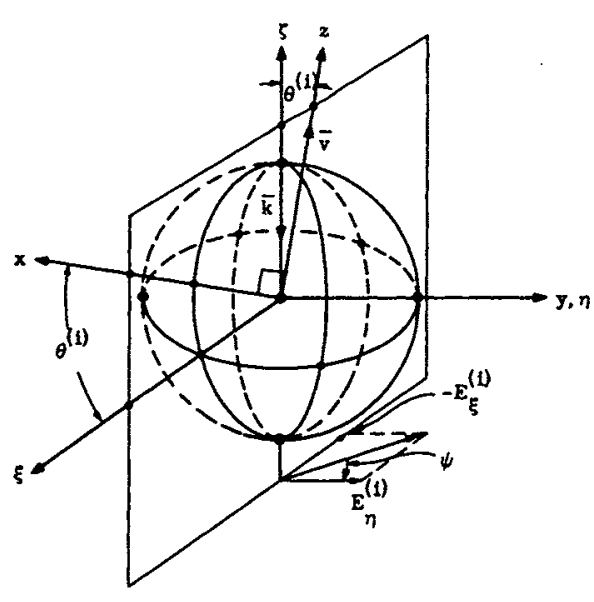

Figure 1. Statement of the problem.

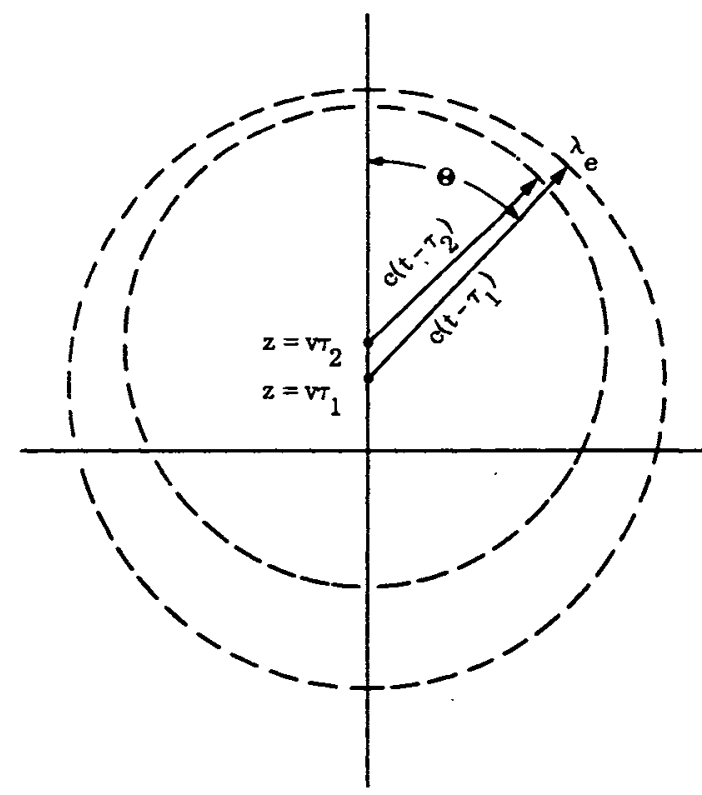

FIGURE 2. Two scattered wave fronts. 
(ii) The $R^{\prime}, \theta^{\prime}, \theta^{\prime}, t^{\prime}$ system and the $x^{\prime}, y^{\prime}, z^{\prime}, t^{\prime}$ system

$$
\begin{aligned}
& z^{\prime}=R^{\prime} \cos \theta^{\prime} \\
& y^{\prime}=R^{\prime} \sin \theta^{\prime} \cos \Phi^{\prime} \\
& x^{\prime}=-R^{\prime} \sin \theta^{\prime} \sin \Phi^{\prime} \\
& t^{\prime}=t^{\prime} .
\end{aligned}
$$

(iii) The $R, \Theta, \Phi, \tau$ system and the $x, y, z, t$ system

$$
\begin{aligned}
& z=R \cos \theta+v \tau \\
& y=R \sin \theta \cos \Phi \\
& x=-R \sin \theta \sin \Phi \\
& t=\frac{R}{c}+\tau .
\end{aligned}
$$

(iv) The $R, \theta, \phi, \tau$ system and the $\xi, \eta, \zeta, t$ system

$$
\begin{aligned}
\zeta & =R \cos \theta+v \tau \cos \theta^{(i)} \\
\eta & =R \sin \theta \cos \phi \\
\xi & =-R \sin \theta \sin \phi-v \tau \sin \theta^{(i)} \\
t & =\frac{R}{c}+\tau .
\end{aligned}
$$

Systems (i) and (ii) are seen to be simply spherical coordinate systems existing in the moving frame, having for their respective polar axes the negative $\bar{k}^{\prime}$ direction and the $\bar{v}$ direction. Systems (iii) and (iv) are also spherical coordinate systems, existing in the rest frame, having for their respective polar axes the $\bar{v}$ direction and the negative $\bar{k}$ direction, but with their origins at the retarded position of the sphere.

The relation between (18) and (19) is a rotation about the common $\eta^{\prime}$ - or $y^{\prime}$ :axes; between (19) and (20) the Lorentz transformation; and between (20) and (21) a rotation about the common $y$ - or $\eta$-axes. This implies that, given an event specified by any one set of the above eight four-dimensional coordinate systems, its respective components in the other seven systems may be determined. Let an event be specified by the $(R, \theta, \phi, \tau)$ system, then its components in the other three spherical systems are given by

$$
\begin{gathered}
\cos \theta=\cos \theta \cos \theta^{(i)}+\sin \theta \sin \theta^{(i)} \sin \phi ; \cos \Phi=\frac{\sin \theta \cos \phi}{\sin \theta} \\
\cos \theta^{\prime}=\frac{\cos \theta-\beta}{1-\beta \cos \theta} ; \Phi^{\prime}=\Phi \\
\cos \theta^{\prime}=\cos \theta^{\prime} \cos \theta^{\prime(i)}-\sin \theta^{\prime} \sin \Phi^{\prime} \sin \theta^{(i)} ; \cos \phi^{\prime}=\frac{\sin \theta^{\prime} \cos \Phi^{\prime}}{\sin \theta^{\prime}}
\end{gathered}
$$


and

$$
R^{\prime}=\frac{1-\beta \cos \theta}{\sqrt{1-\beta^{2}}} R
$$

Both ordered sets of unit vectors, $\hat{R}^{\prime}, \hat{\Theta}^{\prime}, \hat{\Phi}^{\prime}$ and $\hat{R}^{\prime}, \hat{\theta}^{\prime}, \hat{\phi}^{\prime}$, are right-handed orthogonal systems with the $\hat{R}^{\prime}$ vector an element of both sets. This implies that the transformation between the $\hat{\theta}^{\prime}, \hat{\Phi}^{\prime}$ vectors and the $\hat{\theta}^{\prime}, \hat{\phi}^{\prime}$ vectors is a rotation about the common $\hat{R}^{\prime}$ vector. Let $\chi^{\prime}$ be the angle between $\hat{\phi}^{\prime}$ and $\hat{\Phi}^{\prime}$ defined so that

(i) $\cos \chi^{\prime}=\sin \Phi^{\prime} \sin \phi^{\prime}+\cos \Phi^{\prime} \cos \phi^{\prime} \cos \theta^{\prime(l)}$

(ii) $\chi^{\prime}>0$ if a rotation in the right-hand sense about $\hat{R}^{\prime}$ takes $\hat{\phi}^{\prime}$ to $\hat{\Phi}^{\prime}$.

A vector given by its components in spherical system (i) may be resolved into components in spherical system (ii) by

and

$$
E_{\Theta}^{\prime(s)}=E_{\theta}^{\prime(s)} \cos \chi^{\prime}+E_{\phi}^{\prime(s)} \sin \chi^{\prime}
$$

The field may now be resolved into components parallel to and perpendicular to the direction of motion and transformed to the rest frame (Sommerfeld, 1964a). The result, expressed in components in system (iii), is

$$
E(s)=\frac{\sqrt{1-\beta^{2}}}{1-\beta \cos \theta} E_{\Theta}^{\prime(s)}
$$

and

$$
E_{\Phi}^{(s)}=\frac{\sqrt{1-\beta^{2}}}{1-\beta \cos \theta} E_{\Phi}^{\prime(s)}
$$

Finally, the components in this system may be resolved into components in the $(R, \theta, \phi, \tau)$ system given by

and

$$
E_{\theta}^{(s)}=E(g) \cos \chi-E_{\phi}^{(s)} \sin \chi
$$

where $\chi$ satisfies (26) written without the primes.

Equations (7) and (8) may be substituted into (27) to (32) to obtain an expression for the scattered field in the rest frame, which is

$$
\begin{aligned}
& E_{\theta}^{(s)}=-i \frac{E^{(i)}}{2} \frac{1-\beta^{2}}{(1-\beta \cos \theta)^{2}}\left\{\sin \left(\chi-\chi^{\prime}\right) \sin \left(\phi^{\prime}-\psi\right) S_{2}\left(\theta^{\prime}\right)\right. \\
& \left.+\cos \left(\chi-\chi^{\prime}\right) \cos \left(\phi^{\prime}-\psi\right) S_{1}\left(\theta^{\prime}\right)\right\} \frac{e^{-i k \cdot c\left(1+\beta \cos \theta^{(i)}\right)}}{k R}+\text { c.c. }, \\
& E_{\phi}^{(s)}=i \frac{E^{(i)}}{2} \frac{1-\beta^{2}}{(1-\beta \cos \theta)^{2}}\left\{\cos \left(\chi-\chi^{\prime}\right) \sin \left(\phi^{\prime}-\psi\right) S_{2}\left(\theta^{\prime}\right)\right. \\
& \left.-\sin \left(\chi-\chi^{\prime}\right) \cos \left(\phi^{\prime}-\psi\right) S_{1}\left(\theta^{\prime}\right)\right\} \frac{e^{-i k c\left(1+\beta \cos \theta^{(i)}\right)}}{k R}+c . c .,
\end{aligned}
$$


For an observer whose position is fixed, $\theta, \phi$, and $R$ are each functions of $\tau$, which in turn is a function of time. However, the change in $\theta, \phi$, and $R$ is small over several time periods of oscillation and several wavelengths in space if $\beta \ll k R$, which is implied by the far-field condition $l \ll k R$. This implies that the scattered far field appears as a quasi-plane wave whose magnitude, direction of propagation, frequency, wavelength, and polarization are changing with time. The change in frequency is seen to be a first-order in $\beta$ effect, being given by (15), and is the wellknown Doppler shift. The shift in the direction of propagation may alternately be regarded as an aberration due to the motion or the appearance of the sphere in a retarded position due to the finite velocity of propagation of light.

Since the far field for an arbitrary finite object has the same form as (7) and (8), the above results could be generalized by including the appropriate spherical harmonics.

\section{Power and Energy in the Scattered Field}

Since the electromagnetic field is not a harmonic function of time, the concept of average power flow must be investigated. Direct substitution of (33), (34), and (35) shows that the Poynting vector has the form

$$
\left|\bar{E}^{(s)} \times \bar{H}^{(s)}\right|=f(\theta, \phi, R) e^{2 i C r}+f^{*} e^{-2 i C r}+g(\theta, \phi, R),
$$

where the expressions for the resulting constant $C$, the function $f$, and its conjugate $f^{*}$ have not been written out to simplify the discussion. The function $g$ is given below. With the assumption that $\theta, \phi$, and $R$ remain essentially constant over several periods of oscillation and the fact that $\tau$ is an increasing function of time, the average ${ }^{3}$

$$
\begin{aligned}
\left\langle\left|\bar{E}^{(s)} \times \bar{H}^{(s)}\right|\right\rangle & =\lim _{\left(t_{2}-t_{1}\right) \rightarrow \infty} \frac{1}{t_{2}-t_{1}} \int_{t_{1}}^{t_{2}} E^{(s)} \times H^{(s)} d t, \\
& \cong g(\theta, \phi, R)=\frac{I_{0}}{k^{2} R^{2}}\left(\frac{\sqrt{1-\beta^{2}}}{1-\beta \cos \theta}\right)^{4} \cdot\left\{\sin ^{2}\left(\phi^{\prime}-\psi\right) S_{2} S_{2}^{*}+\cos ^{2}\left(\phi^{\prime}-\psi\right) S_{1} S_{1}^{*}\right\} .
\end{aligned}
$$

In (36)

$$
I_{0}=\eta_{0} \frac{E^{(i)} E^{*(i)}}{2} \text { and } \eta_{0}=\sqrt{\frac{\epsilon_{0}}{\mu_{0}}} \text {. }
$$

For the small sphere, low frequency case $\left(k^{\prime} a \ll 1\right)$, (36) may be approximated

$$
\begin{aligned}
\left\langle\bar{E}^{(s)} \times \bar{H}^{(s)}\right\rangle \sim \frac{I_{0} k^{4} a^{6}}{4 R^{2}} \frac{\left(1+\beta \cos \theta^{(i)}\right)^{6}}{\left(1-\beta^{2}\right)(1-\beta \cos \theta)^{4}}\{ & 4-3 \cos ^{2}\left(\phi^{\prime}-\psi\right) \\
& \left.+4 \cos \theta^{\prime}+\left[1+3 \cos ^{2}\left(\phi^{\prime}-\psi\right)\right] \cos ^{2} \theta^{\prime}\right\} R .
\end{aligned}
$$

The scattering cross section is given by (see figs. $3-5$ )

$$
\sigma=\left\langle\left|\frac{\bar{E}^{(s)} \times \bar{H}^{(s)}}{I_{0}}\right|\right\rangle 4 \pi R^{2} .
$$

The total flux of energy, due to the scattered field, out of a large spherical volume $V_{0}$, having

\footnotetext{
${ }^{3}$ The quantities $x$ and $x^{\prime}$ disappear by virtue of the identity $\cos ^{2}\left(x-x^{\prime}\right)+\sin ^{2}\left(x-x^{\prime}\right)=1$
} 


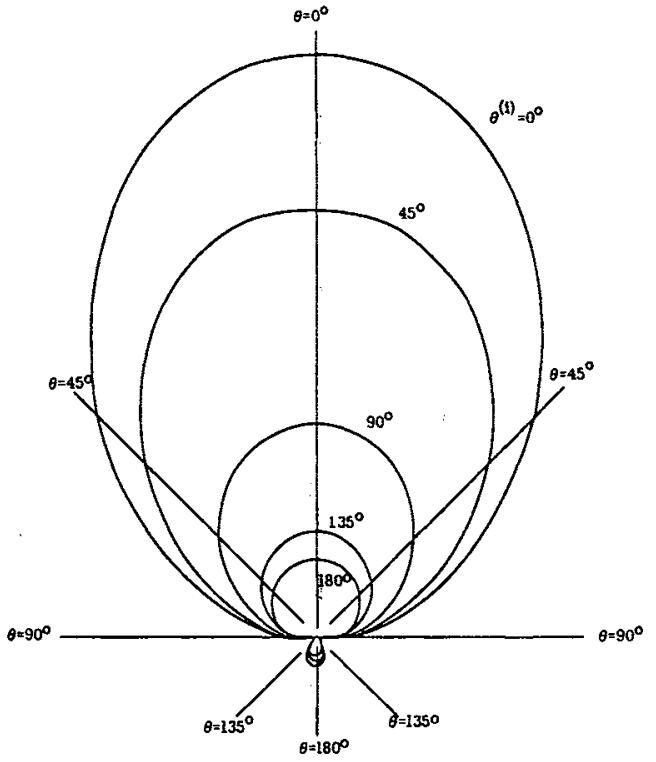

FIGURE 3. $\sigma$ in the $\zeta^{-} \eta$ plane for $\beta=0.1 ; \psi=0$; and several angles of incidence, $\theta^{(i)}$.

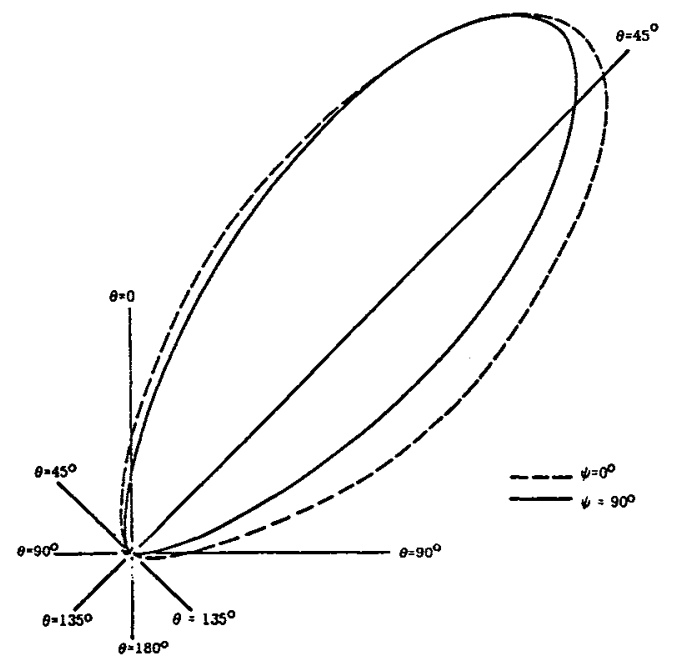

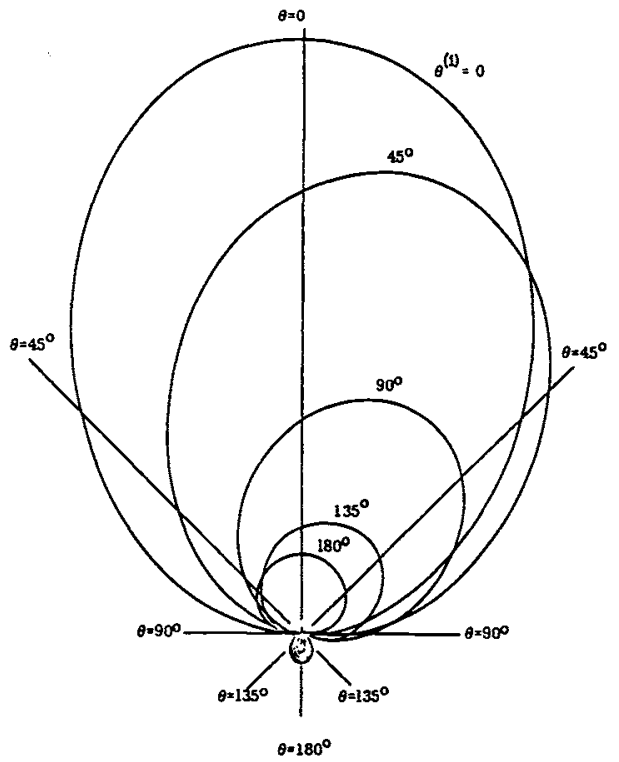

FIGURE 4. $\sigma$ in the $\zeta-\xi$ plane for $\beta=0.1 ; \psi=90^{\circ} ;$ and several angles of incidence, $\theta^{(i)}$.

Figure 5, $\sigma$ in the $\zeta-\xi$ plane for $\beta=0.60$; both polarizations; and $\theta^{(1)}=45^{\circ}$; $\sigma$ ax for $\beta=0.60$ is approximately 200 times $\sigma_{M A X}$ for $\beta=0.00$ (approximately $23 d B$ difference).

a surface area $S_{0}$, containing the conducting sphere may be obtained by direct integration of (36) resulting in

$$
\begin{aligned}
P_{\text {out }}^{(s)}=\int_{S_{0}}<\bar{E}^{(s)} \times \bar{H}^{(s)} & >\cdot d \bar{S}=\frac{\pi I_{0}}{k^{2}\left(1-\beta^{2}\right)}\left\{\left(1+\frac{\beta^{2}}{2} \sin ^{2} \theta^{(i)}\right) I_{1}\right. \\
& \left.+2 \beta \cos \theta^{\prime(i)} I_{2}+\beta^{2}\left(\cos ^{2} \theta^{\prime(i)}-\frac{1}{2} \sin ^{2} \theta^{(i)}\right) I_{3}+\frac{\beta^{2}}{4} \sin ^{2} \theta^{(i)} \cos 2 \psi I_{4}\right\} .
\end{aligned}
$$

The terms $I_{1}, I_{2}, I_{3}$, and $I_{4}$ are presented in the appendix.

It is also possible to calculate the average time rate of change of stored scattered electromagnetic energy, $W^{(s)}$, within the volume $V_{0}$. This is found to be 


$$
\begin{aligned}
\frac{d W^{(s)}}{d t}= & \frac{d}{d t} \iiint_{V_{0}}<\frac{\bar{E}^{(s)} \cdot \bar{E}^{(s)} \epsilon_{0}}{2}+\frac{\bar{B}^{(s)} \cdot \bar{B}^{(s)}}{2 \mu_{0}}>d V \\
= & -\frac{\pi I_{0}}{k^{2}\left(1-\beta^{2}\right)}\left\{\left(\beta^{2}+\frac{\beta^{2}}{2} \sin ^{2} \theta^{\prime(i)}\right) I_{1}+\left(\beta+\beta^{3}\right) \cos \theta^{\prime(i)} I_{2}\right. \\
& \left.+\beta^{2}\left(\cos ^{2} \theta^{\prime(i)}-\frac{1}{2} \sin ^{2} \theta^{\prime(i)}\right) I_{3}+\frac{\beta^{2}}{4} \sin ^{2} \theta^{\prime(i)} \cos 2 \psi I_{4}\right\}
\end{aligned}
$$

The rate of flow of energy from the sphere into the scattered field is

$$
P_{\text {out }}^{(s)}+\frac{d W^{(s)}}{d t}=\frac{\dot{\pi} I_{0}}{k^{2}}\left\{I_{1}+\beta \cos \theta^{(i)} I_{2}\right\}
$$

Unlike the case of scattering by a stationary conducting body, there is an exchange of energy between the electromagnetic field and a moving scattering body. The energy appears as mechanical energy and the exchange process may go in either direction.

The time averaged rate of flow of energy from the field to the sphere may be calculated by integrating the quantity $\bar{E} \cdot \bar{J}$, which is interpreted here as the rate of flow of energy per unit volume from the field to the material medium in which the currents, $\vec{J}$, exist (in this case the conducting sphere) in the following way

$$
P_{m}=\lim _{\left(t_{2}-t_{1}\right){ }_{\infty}} \frac{1}{t_{2}-t_{1}} \int_{t_{1}}^{t_{2}} \int_{-\infty}^{\infty} \int_{-\infty}^{\infty} \int_{-\infty}^{\infty}(\bar{E} \cdot \bar{J}) d x d y d z d t
$$

Now the variables of integration are changed to the primed variables. The Jocobian of the transformation is unity. The integrand, by the Special Theory of Relativity (Sommerfeld, 1964a), $\vec{E} \cdot \bar{J}=\left(\bar{E}^{\prime} \cdot \bar{J}^{\prime}+\bar{v} \cdot \bar{f}^{\prime}\right) / \sqrt{1+\beta^{2}}=v f_{z}^{\prime} / \sqrt{1+\beta^{2}}$ where $\bar{f}$ is the Lorentz force density acting on the body and $f_{z}^{\prime}$, its $z^{\prime}$ component. In the primed system $\bar{E}^{\prime} \cdot \bar{J}^{\prime}=0$ since within the sphere $\bar{E}^{\prime}=0$, on the surface $\bar{E}^{\prime}$ and $\bar{J}^{\prime}$ are perpendicular, and outside of it $\bar{J}^{\prime}=0$. The domain $-\infty<x<\infty$; $-\infty<y<\infty ;-\infty<z<\infty ; t_{1}<t<t_{2}$ maps, under the Lorentz transformation, to $-\infty<x^{\prime}<\infty$; $-\infty<y^{\prime}<\infty ;-\infty<z^{\prime}<\infty ; t_{1} \sqrt{1-\beta^{2}}<t^{\prime}+v z^{\prime} / c^{2}<t_{2} \sqrt{1-\beta^{2}}$. The result is

$$
P_{m}=\lim _{\left(t_{2}-t_{1}\right) \rightarrow \infty} \frac{1}{t_{2}-t_{1}} \int_{-\infty}^{\infty} \int_{-\infty}^{\infty} \int_{-\infty}^{\infty} \int_{t_{1}}^{t_{2} \sqrt{1-\beta^{2}}-v z^{\prime} / c^{2}} \frac{v f_{z}^{\prime}}{\sqrt{1-\beta^{2}}-v z^{\prime} / c^{2}} d t^{\prime} d z^{\prime} d y^{\prime} d x^{\prime}
$$

Since in the primed system the sphere is stationary, the field quantities are harmonic with time and the time integration above may be carried out to give

$$
P_{m}=v\left\langle F_{z}^{\prime}\right\rangle
$$

where $\left\langle F_{z}^{\prime}\right\rangle$ is the time averaged total electromagnetic force acting on the sphere in the $z^{\prime}$ direction. This force may be obtained from Debye's calculation of the light pressure on a sphere (1909) giving finally

$$
P_{m}=-\frac{\beta I_{0} \pi}{k^{2}}\left[I_{5}+I_{2}\right] \cos \theta^{\prime(i)}
$$

where $I_{2}$ and $I_{5}$ are given in the appendix. From (43) and (40) it may be concluded that

$$
\frac{\pi I_{0}}{k^{2}}\left\{I_{1}-\beta I_{5} \cos \theta^{\prime(i)}\right\}
$$

represents the flow of energy from the incident electromagnetic field to the sphere. 


\section{Conclusion}

An expression for the far field scattered by a perfectly conducting sphere moving with uniform velocity through an electromagnetic plane wave has been obtained here. The expression is relativistically correct, valid for arbitrary polarization and direction of propagation of the incident wave, and can be used to calculate the scattered electromagnetic field at any position and time provided only that the far-field condition is fulfilled. The scattered wave appears locally that of a plane wave with changing direction of propagation, frequency, polarization, and magnitude, exhibiting the aberration of light effect and the Doppler effect.

The solution was seen to lend itself to the calculation of the various forms of energy. It is also valid for an arbitrarily shaped, uniformly moving, finite body whose stationary solution is known.

The author thanks Professor Chen-To Tai for his valuable suggestions and advice. Parts of this paper were presented at the 1967 Spring URSI meeting in Ottawa, Ontario, Canada, by R. C. Restrick and Chen-To Tai.

\section{Appendix}

$$
\begin{aligned}
I_{1} & =\int_{0}^{\pi}\left[S_{1}\left(\theta^{\prime}\right) S_{1}^{*}\left(\theta^{\prime}\right)+S_{2}\left(\theta^{\prime}\right) S_{2}^{*}\left(\theta^{\prime}\right)\right] \sin \theta^{\prime} d \theta^{\prime} \\
& =2 \sum_{n=1}^{\infty}(2 n+1)\left(\alpha_{n}^{*} \alpha_{n}+\beta_{n} \beta_{n}^{*}\right) \\
I_{2} & =\int_{0}^{\pi}\left[S_{1}\left(\theta^{\prime}\right) S_{1}^{*}\left(\theta^{\prime}\right)+S_{2}\left(\theta^{\prime}\right) S_{2}^{*}\left(\theta^{\prime}\right)\right] \cos \theta^{\prime} \sin \theta^{\prime} d \theta^{\prime} \\
& =-2 \sum_{n=1}^{\infty}\left\{\left(\alpha_{n}^{*} \beta_{n}+\text { c.c. }\right) \frac{2 n+1}{n(n+1)}+\left(\alpha_{n+1}^{*} \alpha_{n}+\beta_{n+1} \beta_{n}^{*}+\text { c.c. }\right) \frac{n(n+2)}{n+1}\right\} ;
\end{aligned}
$$

$$
I_{3}=\int_{0}^{\pi}\left[S_{1}\left(\theta^{\prime}\right) S_{1}^{*}\left(\theta^{\prime}\right)+S_{2}\left(\theta^{\prime}\right) S_{2}^{*}\left(\theta^{\prime}\right)\right] \cos ^{2} \theta^{\prime} \sin \theta^{\prime} d \theta^{\prime}
$$$$
=2 \sum_{n=1}^{\infty}\left\{\left(\alpha_{n}^{*} \alpha_{n+2}+\beta_{n+2} \beta_{n}^{*}+\text { c.c. }\right) \frac{n(n+3)}{2 n+3}\right.
$$$$
\left.+\left(\alpha_{n}^{*} \beta_{n+1}+\alpha_{n+1} \beta_{n}^{*}+\text { c.c. }\right) \frac{2}{n+1}+\left(\alpha_{n}^{*} \alpha_{n}+\beta_{n} \beta_{n}^{*}\right)(2 n+1) \frac{(2 n+1)^{4}-12(2 n+1)^{2}+59}{8 n(n+1)(2 n+3)(2 n-1)}\right\} ;
$$

$$
\begin{aligned}
& I_{4}=\int_{0}^{\pi}\left[S_{2}\left(\theta^{\prime}\right) S_{2}^{*}\left(\theta^{\prime}\right)-S_{1}\left(\theta^{\prime}\right) S_{1}^{*}\left(\theta^{\prime}\right)\right] \sin ^{3} \theta^{\prime} d \theta^{\prime} \\
& \begin{aligned}
=\sum_{n=1}^{\infty}\left(\alpha_{n} \alpha_{n}^{*}-\beta_{n} \beta_{n}^{*}\right)(2 n+1) \frac{(2 n+1)^{2}-13}{(2 n+1)^{2}-4} \\
\quad-2\left(\alpha_{n} \alpha_{n+2}^{*}-\beta_{n} \beta_{n+2}^{*}+\text { c.c. }\right) \frac{n(n+3)}{2 n+3}+4\left(\alpha_{n} \beta_{n+1}^{*}-\beta_{n} \alpha_{n+1}^{*}+\text { c.c. }\right) ;
\end{aligned}
\end{aligned}
$$

$I_{5}=\sum_{1}^{\infty}(2 n+1)\left(\alpha_{n}+\beta_{n}+c . c.\right)$. 


\title{
9. References
}

Courant, R., and D. Hilbert (1962), Methods of Mathematical Physics, II (Interscience Publ., Inc., New York, N.Y.).

Debye, P. (1909), Der Lichtdruck auf Kugeln von beliebigem Material, Ann. Physik 30, 57-136.

Lee, S. W., and Y. T. Lo (1967), Reflection and transmission of electromagnetic waves by a moving uniaxially anisotropic medium, J. Appl. Phys. 38, No. 2, 870-875.

Lee, S. W., and R. Mittra (1967), Scattering of electromagnetic waves by a moving cylinder in free space, Can. J. Phys. 45, No. 9, 2999-3007.

Lewis, R. M., and W. Pressman (1966), Radiation and propagation of electromagnetic waves from moving sources, Radio

Sci. 1 (New Series), No. 9, 1029-1040.

Pauli, W. (1958), Theory of Relativity (Pergamon Press, New York, N.Y.).

Pyati, V. (1966), Radiation due to an infinite oscillating dipole over a lossless semi-infinite moving dielectric medium, Univ.

Michigan, Radiation Lab. Rept. No. 7322-2-T.

Sommerfeld, A. (1964a), Electrodynamics; Lectures on Theoretical Physics, III (Academic Press, New York, N.Y.).

Sommerfeld, A. (1964b), Optics; Lectures on Theoretical Physics, IV (Academic Press, New York, N.Y.).

Tai, C-T. (1965), Two boundary-value problems involving moving media, presented at URSI Spring meeting, Washington, D.C.

Yeh, C. (1965), Reflection and transmission of electromagnetic waves by a moving dielectric medium, J. Appl. Phys. 36, No. $11,3513-3517$.

(Paper 3-12-480)

\section{Scattering of Electromagnetic Waves From a Slightly Rough Surface Moving With Uniform Velocity}

\author{
G. R. Valenzuela \\ Naval Research Laboratory, Washington, D.C. 20390, U.S.A. \\ (Received May 14, 1968; revised July 5, 1968)
}

\begin{abstract}
The scattering of electromagnetic waves from a slightly rough surface moving with uniform velocity is investigated.

Rice's generalization of Rayleigh's expansion for the scattered fields and Lorentz transformation are used to relate the fields in the moving and stationary reference frames. In the development we also use the principle of phase invariance and the covariance nature of the electromagnetic fields.
\end{abstract}

\section{Introduction}

In theoretical studies dealing with the scattering of electromagnetic waves from the sea surface, Doppler effects encountered in radar sea-echoes have not been treated. On the experimental side, there are a few investigations performed with coherent radars. Among them, Crombie (1955) observed that the Doppler frequency for sea-echo at a frequency of $13.56 \mathrm{MHz}$, was surprisingly constant regardless of sea conditions and had a magnitude which corresponded to the phase velocity of gravity waves of a period equal to half the

'This paper was presented at the 1968 URSI Spring meeting, Washington, D.C. wavelength of the electromagnetic radiation. This is a characteristic of slightly rough surface scattering. From the measured Doppler spectra, he concluded that the return could be explained in terms of trains of waves and not by a continuous wave (thus he found the sea is not homogeneous). Hicks et al (1960) obtained with an $X$-band radar, horizontally polarized, that sea-echoes seemed to come from two sets of scatterers: a set of sea waves of unbroken water, of a period of one-half the wavelength of the radar frequency (slightly rough surface scattering), that produced a strong and symmetrical Doppler spectrum with a mean Doppler frequency equivalent to the particle motion of larger sea waves and a second set of scatterers, of wind driven 